\title{
Earth-Life Transitions: Towards a New Understanding of Earth Systems
}

\author{
EarthRates RCN ${ }^{1}$ \\ ${ }^{1}$ University of Connecticut
}

May 5, 2020

Lyons, T., Hren, M., Fox, D., Hyland, E., and Kehrwald, N.. (2018) Earth-Life Transitions: Towards a

New Understanding of Earth Systems. EarthRates, Minneapolis, MN. https://drive.google.com/file/ d/1Yo6SclBfS_YPWg8JJWKQ3rKbgRnOqKOm/view?usp=sharing 